
\title{
25 Research Square \\ HBx Inhibits DNA Sensing Signaling Pathway via Ubiquitination and Autophagy of cGAS
}

\section{Hong Chen}

Chongqing Medical University

\section{Linshan Jiang}

Chongqing Medical University

\section{Shu Chen}

Chongqing Medical University

Qin Hu

Chongqing Medical University

\section{Ying Huang}

Chongqing Medical University

\section{Ying Wu}

Guangxi Medical University

\section{Weixian Chen (D300801@cqmu.edu.cn )}

Chongqing Medical University https://orcid.org/0000-0001-6973-9338

\section{Research Article}

Keywords: cGAS, HBx, IFN- $\beta$, ubiquitination, autophagy

Posted Date: February 4th, 2022

DOI: https://doi.org/10.21203/rs.3.rs-1238333/v1

License: (c) (i) This work is licensed under a Creative Commons Attribution 4.0 International License.

Read Full License

Version of Record: A version of this preprint was published at Virology Journal on March 28th, 2022. See the published version at https://doi.org/10.1186/s12985-022-01785-3. 


\section{Abstract}

(1) Background: Cyclic GMP-AMP synthase (cGAS) is a crucial DNA sensor and plays an important role in host antiviral innate immune responses. During hepatitis B virus (HBV) infection, the cGAS signaling pathway can be activated to suppress HBV replication. As an important regulatory protein of HBV, hepatitis $B$ virus $X$ protein $(\mathrm{HBX})$ may serve as an antagonistic character to the cGAS/STING signaling pathway. In this study, we aim to investigate the functional role of HBx in the cGAS/STING signaling pathway.

(2) Methods: The effects of HBx on IFN- $\beta$ promoter activity was measured by Dual-luciferase reporter assays. Ubiquitination and autophagy were analyzed by Western-blot and Co-immunoprecipitation assays.

(3) Results: Our results shown that HBx down-regulates IFN-I production by directly promoting ubiquitination and autophagy degradation of cGAS.

(4) Conclusions: HBV can antagonize host cGAS DNA sensing to promote HBV replication and provide novel insights to develop novel approaches against HBV infection.

\section{Introduction}

The innate immune system constitutes the first line of host defense against invading pathogens and recognizes pathogen-associated molecular patterns through interacting with various pathogen recognition receptors (PRRs), then triggering the production of type I interferon (IFN-I), tumor necrosis factor, and other antiviral factors [1],[2]. cGAS serves as a cytosolic DNA sensor belonging to the PRR, which catalyzes the synthesis of CGAMP and induces the activation of transcription factor interferon regulatory factor-3 (IRF-3), subsequently, promotes IFN-I release and IFN stimulated genes (ISGs) expression such as ISG15, ISG56 [3, 4]. Besides, cGAS exhibits antiviral activity against a series of DNA and RNA viruses, such as herpes simplex virus 1 (HSV-1), dengue virus (DENV), and HIV [5-7]. Therefore, it is widely accepted that cGAS is involved in the recognition of HBV. Importantly, growing evidence indicates that the cGAS-mediated signaling pathway promotes innate immune response against HBV.

Furthermore, several research groups reported that HBV replication could be inhibited by cGAS. In brief, cGAS is shown to recognize HBV DNA and is required to initiate the antiviral innate immunity to suppress HBV replication [8-10]. Since activation of cGAS elicits a potent antiviral response, HBV may enable its maintenance in infected hepatocytes by employing strategies to attenuate the cGAS-STING signaling cascade.

HSV-1 tegument protein VP24, UL41, UL24, UL46, UL36, US3, and VP22 were demonstrated to evade the cGAS/STING-mediated signaling pathway[11-17]. On the other hand, Kaposi's sarcoma-associated herpesvirus (KSHV) ORF52 suppresses cytosolic DNA sensing by directly inhibiting cGAS enzymatic activity through cGAS binding and DNA binding[18]. KSHV's N-terminal domain of the latency-associated 
nuclear antigen (LANA) interacted directly with cGAS, thereby antagonizing cGAS and inhibiting the subsequent 2'3' cGAMP and IFN-I production[19]. However, the underlying mechanisms of HBV evading innate immune responses are still poorly understood. cGAS could sense the naked relaxed-circular HBV DNA but fail to sense the nucleic acids during HBV infection as the genome is likely packaged to the viral capsid. Nevertheless, it is reported that HBV infection suppresses the expression and function of cGAS [20]. However, studies are needed to elucidate the potential specific mechanisms responsible for suppressing cGAS by HBV.

HBV is a hepatotropic, non-cytopathic, enveloped, and partially double-stranded DNA (dsDNA) (3.2 kb) virus classified into the Hepadnaviridae family[21] which can be sensed by the cGAS/STING signaling pathway and activate the host antiviral responses. HBx is the unique non-structure protein of HBV and encoded by one of the four open reading frames of HBV. It is well known as an important coactivator for HBV replication and HBV-associated hepatocellular carcinogenesis [22]. HBV is also regarded as a stealth virus because it could invade and replicate efficiently in the human liver by escaping from the host's innate antiviral immunity. Recently, a novel viral strategy has been reported that HBV suppresses the expression and function of cGAS to evasion from the innate immune responses activated by the cGAS/STING signaling pathway[20]. However, the specific mechanisms involving HBV inhibiting the expression and function of cGAS have not been fully illustrated. We report here that HBx, as an HBVspecific component protein, downregulates the accumulation of cGAS expression through promoting its ubiquitination and autophagy, thereby escaping the innate immune response activated by the cGAS/STING signaling pathway and establishing an effective infection

\section{Methods}

\section{Cell culture and transfection}

The human hepatocellular carcinoma cell lines SMMC-7721, LO2, HepG2, HepG2.2.15, and HEK293T, were maintained in the Dulbecco's modified Eagle's medium (DMEM) with 10\% FBS (Gibco, USA), 100 $\mathrm{U} / \mathrm{ml}$ penicillin, and $100 \mu \mathrm{g} / \mathrm{ml}$ streptomycin. The cell culture was maintained at $37^{\circ} \mathrm{C}$ in a humid atmosphere containing 5\% CO2. Transient transfections were performed with Lipofectamine 2000 according to the manufacturer's protocol.

\section{Reagents and antibodies}

The primary antibodies used for this study were as follows: mouse anti-HBx monoclonal antibody (Santa Cruz, USA), rabbit anti-cGAS monoclonal antibody (CST, USA), rabbit anti-STING monoclonal antibody (CST, USA), rabbit anti-IRF3(Phospho-Ser382) monoclonal antibody (CST, USA), mouse anti-IRF3 monoclonal antibody (CST, USA), mouse anti- $\beta$-actin monoclonal antibody (Santa Cruz, USA), rabbit antiFlag polyclonal antibody (Proteintech, USA), rabbit anti-HA polyclonal antibody (Proteintech, USA), K48linked ubiquitin antibody(Billerica, MA, USA), DAPI (Santa Cruz, USA) and Lipofectamine 2000 (Invitrogen, USA) were used following the manufacturer's instructions. 


\section{Luciferase Reporter Assays}

HEK293T cells seeded on 24-well plates were transiently transfected with $200 \mathrm{ng}$ of the luciferase reporter plasmid together with a total of $600 \mathrm{ng}$ of various expression plasmids and/or empty vector controls using Lipofectamine 2000. As an internal control, $50 \mathrm{ng}$ of pRL-TK (Renilla luciferase) was transfected simultaneously. Luciferase assays were then performed at $24 \mathrm{~h}$ post-transfection according to the Promega Dual-Luciferase Reporter Assay protocol. The relative luciferase activity was expressed as arbitrary units by normalizing firefly to Renilla luciferase activity.

\section{Real-time quantitative PCR analysis}

Cells were transfected for $24 \mathrm{~h}$ and then lysed with Trizol (Invitrogen, Carlsbad, CA, USA). Complementary single-stranded DNA was synthesized from total RNA by reverse transcription (TaKaRa, Japan).

Quantification of cDNA targets was performed on CFX96TM Real-Time-PCR Detection System (Bio-Rad, USA). Primers were synthesized by Invitrogen and are listed in Table 1.

\section{Western blotting}

The levels of cGAS, STING, HBx, and IRF3 in cells were evaluated by western blotting. Briefly, samples containing an equal amount of protein were separated by SDS-PAGE and blotted onto PVDF membranes. The membranes were blocked with $5 \%$ bovine serum albumin and incubated with anti-cGAS, anti-STING, anti-HBx, anti-IRF3, anti-Flag, anti-HA, anti-K48-linked ubiquitin, or anti-ß-actin antibodies, following by incubation with secondary antibodies conjugated with horseradish peroxidase. The proteins of interest were detected using the SuperSignal West Pico Chemiluminescent Substrate kit (Thermo Fisher Scientific, USA). The results were recorded by the Bio-Rad Electrophoresis Documentation (Gel Doc 1000, Bio-Rad, USA) and Quantity One Version 4.5.0.

\section{Native PAGE}

Native polyacrylamide gel electrophoresis (PAGE) was performed using ReadyGels (7.5\%, Bio-Rad). In brief, gels were prerun with $25 \mathrm{mM}$ Tris and $192 \mathrm{mM}$ glycine, $\mathrm{pH} 8.4$, with $1 \%$ deoxycholate (DOC) in the cathode chamber for $30 \mathrm{~min}$ at $40 \mathrm{~mA}$. Samples in native sample buffer $(10 \mathrm{~g}$ of protein, $62.5 \mathrm{mM}$ Tris-Cl, $\mathrm{pH} 6.8,15 \%$ glycerol, and 1\% DOC) were size-fractionated by electrophoresis at $25 \mathrm{~mA}$, and the gel was transferred to nitrocellulose membranes for WB analysis.

\section{Statistical analysis}

The Student's t-test was used for all statistical analyses with GraphPad Prism 5.0 software(GraphPad Software, CA, USA). Differences between groups were considered significant when the P-value was less than 0.05. Statistical differences were showed to be at different levels of $\mathrm{P}<0.05(*)$ and $\mathrm{P}<0.001$ $(* * *)$.

\section{Results}




\section{Hepatitis $B$ virus $X$ protein is a negative regulator of the cGAS-induced IFN-I signaling pathway}

Currently, substantial evidence demonstrates that cGAS recognizes cytosolic DNA, leading to IFN-I production. $\mathrm{HBx}$ is an enigmatic molecule because of its pleiotropic functions in regulating IFN-I production[23]. To evaluate the effect of HBx on the cGAS/STING signaling pathway during DNA virus infection, we carried out dual-luciferase reporter (DLR) assays to detect interferon beta (IFN- $\beta$ ) promoter activity. In HEK293T cells, transfection with cGAS or a minimal amount of STING alone could not activate the IFN- $\beta$ promoter, whereas cotransfection of $c$ GAS and STING plasmids significantly activated the IFN- $\beta$ promoter (Figure 1a). Then, HEK293 cells were cotransfected with cGAS, STING, and IFN- $\beta$ luciferase reporter in the presence or absence of HBx. The results showed that HBx could significantly inhibit the IFN- $\beta$ promoter activity in a dose-dependent manner (Figure. 1b, c). We further measured the mRNA of IFN- $\beta$ to validate the expression level, and similar results were obtained (Figure. $1 \mathrm{~d}$ ).

Next, cGAS and STING expression levels were detected in several hepatic cell lines, including HepG2, HepG2.2.15, L02, and SMMC-7721. The results indicated that STING was highly expressed in all the cell lines. However, we only detected the expression of cGAS in L02 and SMMC-7721 cells (Figure. 1e).

It is reported that cGAS senses cytosolic DNA and catalyzes the synthesis of cGAMP, which subsequently binds to STING, leading to IRF3 dimerization and phosphorylation, finally producing IFN- $\beta[3,24]$. Previous studies have reported that HBx could suppress virus-triggered IRF3 activation through the act on MAVS to inhibit the expression of IFN- $\beta$ [25]. Dimerization and phosphorylation are the activated state of IRF3, and we verified that HBx could suppress cGAS-triggered dimerization and phosphorylation of IRF3 to inhibit IFN- $\beta$ (Figure. 1f, g).

\section{HBX inhibits the IFN-I signaling pathway upstream of STING.}

To clarify what level HBx acts on the CGAS-STING pathway to block the production of IFN- $\beta$, we combined HEK293T cells with empty vectors or HBx plasmids, as well as IFN- $\beta$-Luc reporter genes and important adaptor proteins in the expression pathway (including the active forms of STING, tank-binding kinase 1 (TBK1) and IRF3 (IRF3/5D). We found that ectopic expression of HBx does not affect the activation of IFN- $\beta$ promoters driven by STING, TBK1, or IRF3/5D (Figure 2). These results suggest that HBx can inhibit the expression of IFN-I- $\beta$ upstream of STING.

\section{HBx inhibits the cGAS protein level.}

The data mentioned above led us to hypothesize that HBx might mainly act directly on cGAS. KSHV virion protein ORF52 inhibits cGAS enzymatic activity [18]. HSV-1 tegument protein UL41 downregulates the expression of cGAS, and VP22 interacts with cGAS directly to interfere with its DNA sensing $[12,17]$. Previous research has shown that HBV infection suppresses the expression of cGAS and its related genes [20]. HEK293T cell line was used as a cell model to explore whether the role of HBx is consistent with HBV and if $\mathrm{HBx}$ is the active component that directly targets the cGAS. Ectopic expression of cGAS in 
HEK293T was performed to test whether the HBx could decrease the expression level of cGAS. HEK293T cells were ectopically expressed cGAS, and cells were harvested and subjected to Western blot analysis. As shown in Figure.3a, HBx down regulated the expression of cGAS in a dose-dependent manner (Figure.3a). Previous experiments have shown that SMMC-7721 cells contain endogenous cGAS.SMMC7721 cells were transfected with HBx plasmid, harvested, and subjected to Western blot (WB) analysis. As shown in Figure.3b, HBx significantly downregulated the expression of endogenous cGAS (Figure.3b). Interestingly, the cGAS protein level decreased markedly when HBx expression increased. However, the abundance of cGAS mRNA did not change with the increased expression of HBX (Figure.3c). Notably, we observed that HBx did not affect the transcriptional level of cGAS. Further study is required for the regulation mechanism of HBx acting on cGAS.

\section{HBx binds to and interacts with cGAS.}

It is known that DNA binds to the $\mathrm{N}$ terminus of cGAS to promote cGAS activation [24]. In gammaherpesviruses, ORF52 blocks cGAS activity in part through their interaction. The specific inhibition of cGAS by ORF52 prompted us to investigate the possibility of an interaction between cGAS and HBX. Previous reports have revealed that $\mathrm{HBx}$ inhibited the IFN- $\beta$ signaling pathway by decreasing the expression and function of cGAS. To further explore the role of HBx in IFN- $\beta$ production by downregulating the expression of CGAS, SMMC-7721 and LO2 cells were transfected with HBx plasmids, $24 \mathrm{~h}$ after transfection, cells were lysed, and coimmunoprecipitation experiments were performed. Expression of the transfected proteins was analyzed by immunoblotting with STING-HA and cGAS -Flag antibodies. As shown in Figure. 4a, HBx bound and interacted with cGAS but not STING.

Meanwhile, HBx was used to pull down cGAS, and a similar result was observed (Figure. 4b). Then fluorescence microscopy also indicated that HBx co-localized with cGAS in various cell types (Figure. 4c). These results suggest that $\mathrm{HBx}$ binds to cGAS, which may block DNA recognition by cGAS.

\section{HBx promotes ubiquitination and autophagy degradation of cGAS.}

The degradation of protein mainly consists of two pathways: ubiquitin-proteasome and autophagylysosome. It is known that HBx has been shown to play a critical role in HBV-mediated autophagy. It is reported that the lysine 48 (K48)-linked ubiquitin chains of cGAS connect with microtubule-associated protein-Light-chain 3 (LC3), which mediate the autophagic degradation of cGAS [26]. Pharmacologic approaches were employed to investigate which pathways participate in regulating the expression of cGAS. We observed that the autophagic enhancer Rapamycin and HBx down-regulated the protein level of cGAS. Besides, both autophagic inhibitor 3-methyladenine (3-MA) and proteasome inhibitor MG132 could up-regulate the protein level of cGAS (Figure. 5a, b).

LC3 from a diffuse cytoplasmic distribution to point aggregation has been used as a marker of autophagy activation [26]. GFP-LC3 fluorescent particles were detected to reflect the autophagy level. We observed that HBx and Rapamycin dramatically increased GFP-LC3 redistribution into aggregation dots, and 3-MA could suppress this phenomenon. The percentage of GFP-LC3-positive cells induced by HBX 
was increased almost 3-fold compared to the control (Figure. 5c). Besides, (LC3) transforms from a free form (LC3-I) to a phosphatidylethanolamine-conjugated form (LC3-II) during autophagy. LC3-II also serves as an indicator of autophagy [26]. Our study found that HBx and Rapamycin markedly up-regulated LC3-II expression and reversed the ratios of LC3-I/LC3-II compared to the control (Figure. 5d). Previous studies have shown that CGAS was mainly ubiquitinated with K48 linkage. We also detected the K48-linked ubiquitination of cGAS and found that HBx could promote the K48-linked ubiquitination of cGAS (Figure. $5 e)$. To further verify that HBx is the main component protein that regulates the cGAS/STING pathway of HBV infection, ISD is used to sensitized the CGAS/STING signal channel, HBV 1.3-fold genome plasmid (pcDNA-HBV1.3) were transfected into human hepatic cell line L02 to establish an HBV-infected hepatic cell model to detect the downstream products mediated by the cGAS/STING pathway, including IFN- $\beta$, ISG 54, ISG56 expression level. We found that after transfection with HBV1.3, the mRNA levels of IFN- $\beta$, ISG54, and ISG56 all decreased significantly (Figure $5 f, 5 \mathrm{~g}, 5 \mathrm{~h}$ ), comparable with the effect of HBx, suggesting that $\mathrm{HBx}$ is responsible for HBV to inhibit CGAS/STING signaling pathway. These results showed that $\mathrm{HBx}$ as the main component protein of HBV could down-regulate the protein level of cGAS by promoting autophagy and ubiquitination, further inhibiting CGAS mediated pathways, thereby inhibiting the expression of IFN-I- $\beta$ and IFN stimulated genes, such as ISG56, ISG54.

\section{Discussion}

Two main hepatitis viruses currently cause chronic viral hepatitis, including HCV and HBV. HCV is a single-stranded RNA virus, and the treatment of HCV infection has been overcome. However, HBV is so sneaky that the cure for HBV infection is still limited[27]. HBV is a hepatotropic DNA virus [21]. It is widely accepted that DNA sensors bridge the host sensing DNA virus and inducing immune defense during the past decade. HBV infection can be sensed by the DNA sensor of cGAS, which activates the cGAS/STING signaling pathway and induces the innate immune response [9]. HBV is so sly that it prompts us to presume HBV might evolve certain mechanisms to block this signal pathway. cGAS inhibits HBV is widely reported, but the immune evasion of HBV is poorly understood.

There are three main ways to regulate cGAS: (i) post-translational modifications, including phosphorylation, ubiquitination, sumoylation, and glutamylation; (ii) crosstalk with other pathways such as autophagy, inflammasome; (iii) regulation of cGAS by viral proteins [28]. There is growing evidence that various viruses regulate cGAS through these pathways, thereby regulates innate immune response induced by the cGAS signal axis. Kaposi's sarcoma-associated herpesvirus ORF52, HSV-1 tegument protein UL41, VP22, and the N-terminal domain of the latency-associated nuclear antigen was reported to target cGAS directly. Previous studies have shown that cGAS inhibits HBV by multiple strategies. Meanwhile, HBV evades cGAS sensing by decreasing cGAS and its effector gene expression. However, little is known about whether HBV evades the cGAS-STING signaling pathway. For the first time, we reported that HBX is an effective HBV protein targeting cGAS, as HBX could down-regulate cGAS expression and further inhibit IFN- $\beta$ and ISG56 expression induced by cGAS. However, HBX did not downregulate cGAS mRNA, indicating that it did not mediate the transcriptional level of cGAS. 
Ubiquitin-proteasome and autophagy-lysosome are two main regulatory pathways for protein degradation. In HSV-1 infection, TRIM14 recruits USP14 to cleave K48-linked ubiquitination of cGAS at K414 [26]. Through pharmacological research, we confirmed that HBX down-regulated the protein level of cGAS by autophagy and K48-linked ubiquitination, which leads to a weakening of the cGAS signaling waterfall, and in turn, helps HBV escape the innate immune response.

Different research groups have demonstrated in many ways that cGAS is a highly effective DNA sensor. The purified cGAS protein can directly bind to DNA molecules in biochemical tests [3]. Protein crystal structure analysis shows that cGAS binds to negatively charged DNA phosphate skeletons through positive and hydrogen bonding on the protein surface, which does not depend on DNA sequences [29]. Fluorescence co-localization found that cGAS and HBx co-localized intracellularly, and co-IP confirmed that HBx interacts with cGAS but not STING. HBx binds to cGAS, which may interfere with DNA recognition by CGAS. On the one hand, this confirms that $\mathrm{HBx}$ is the meritorious statesman of $\mathrm{HBV}$ in regulating and escaping cGAS-induced innate immunity. On the other hand, it is reasonable to presume that HBx may block cGAS's DNA recognizing.

In summary, Our research reveals that HBx is an effective molecule of HBV regulating cGAS. It remains unknown that whether other pathways or other protein components are involved in HBV regulating cGAS. In addition, we did not investigate the specific sites of GAS binding to HBx and the direct effects caused by their binding, nor did we clarify whether there was any correlation between autophagy and ubiquitination regulation of cGAS. Thus, further studies are still required to elucidate the detailed mechanisms. cGAS debuted as a cytoplasmic DNA sensor. However, recent studies have shown that cGAS is localized mostly in the nucleus [30].

Moreover, cGAS combined with chromatin acts as a decelerator for DNA replication forks, which controls replication dynamics and suppresses replication-associated DNA damage, suggesting that cGAS is an attractive target [31]. cGAS combined with chromatin for exploiting the genomic instability of cancer cells. Whether the cGAS combined with chromatin will interact with the nucleated HBV virus is also unknown. Further investigation of these questions will certainly shed new light on our understanding of the function of cGAS, which will also help us develop novel therapeutic methods against HBV infection.

\section{Conclusion}

In conclusion, in this present study we identified that HBx is an effective molecule of HBV regulatingcGAS. It negatively regulate CGAS-mediated DNA signaling through ubiquitination and autophagy. Studying the role of cGAS in HBV infection provides some new ideas for improving the future treatment of HBV.

\section{Declarations}

\section{Ethics approval and consent to participate}


Not applicable.

\section{Consent for publication}

All authors have agreed on the consent of the manuscript.

\section{Availability of data and materials}

The data and material generated or analyzed in this study are available upon reasonable request, and could be provided by Weixian Chen(300801@cqmu.edu.cn).

\section{Competing interests}

The authors declare that there is no conflict of interest regarding the publication of this article.

\section{Authors contributions}

CWX and WY contributed to conception and design of the study. $\mathrm{CH}$ and JLS performed the experiment, as well as analyzed the data, drafted the manuscript. CS, HQ and HY analyzed the data. All authors contributed to manuscript revision, read, and approved the submitted version.

\section{Funding}

The present study was supported by grants from the National Natural Science Foundation of China (No. 81873971 ) and the Science and Technology Commission Foundation of Chongqing (No. Cstc2016jcyA0264).

\section{Acknowledgments}

We are grateful to Professor Chunfu Zheng for the STING-HA plasmid, IFN-I- $\beta$-Luc plasmid, and IRF3/5DFLAG plasmid. We also would like to thank Professor Fanxiu Zhu for the cGAS-Flag plasmid.

\section{References}

1. Takeuchi O, Akira S. Pattern recognition receptors and inflammation. Cell. 2010;140(6):805-20.

2. Schneider W, Chevillotte M, Rice C. Interferon-stimulated genes: a complex web of host defenses. Annu Rev Immunol. 2014;32:513-45.

3. Sun L, Wu J, Du F, Chen X, Chen Z. Cyclic GMP-AMP synthase is a cytosolic DNA sensor that activates the type I interferon pathway. Science. 2013;339(6121):786-91. 
4. Kato K, Omura H, Ishitani R, Nureki O. Cyclic GMP-AMP as an Endogenous Second Messenger in Innate Immune Signaling by Cytosolic DNA. Annual review of biochemistry. 2017;86:541-66.

5. Diner B, Lum K, Toettcher J, Cristea I. Viral DNA Sensors IFI16 and Cyclic GMP-AMP Synthase Possess Distinct Functions in Regulating Viral Gene Expression, Immune Defenses, and Apoptotic Responses during Herpesvirus Infection. mBio 2016, 7(6).

6. Kao Y, Lai M, Yu C. How Dengue Virus Circumvents Innate Immunity. Frontiers in immunology. 2018;9:2860.

7. Gao D, Wu J, Wu Y, Du F, Aroh C, Yan N, Sun L, Chen Z. Cyclic GMP-AMP synthase is an innate immune sensor of HIV and other retroviruses. Science. 2013;341(6148):903-6.

8. Dansako H, Ueda Y, Okumura N, Satoh S, Sugiyama M, Mizokami M, Ikeda M, Kato N. The cyclic GMP-AMP synthetase-STING signaling pathway is required for both the innate immune response against HBV and the suppression of HBV assembly. FEBS J. 2016;283(1):144-56.

9. He J, Hao R, Liu D, Liu X, Wu S, Guo S, Wang Y, Tien P, Guo D. Inhibition of hepatitis B virus replication by activation of the cGAS-STING pathway. J Gen Virol. 2016;97(12):3368-78.

10. Guo F, Tang L, Shu S, Sehgal M, Sheraz M, Liu B, Zhao Q, Cheng J, Zhao X, Zhou T, et al: Activation of Stimulator of Interferon Genes in Hepatocytes Suppresses the Replication of Hepatitis B Virus. Antimicrobial agents and chemotherapy 2017, 61(10).

11. Zhang D, Su C, Zheng C. Herpes Simplex Virus 1 Serine Protease VP24 Blocks the DNA-Sensing Signal Pathway by Abrogating Activation of Interferon Regulatory Factor 3. Journal of virology. 2016;90(12):5824-9.

12. Su C, Zheng C. Herpes Simplex Virus 1 Abrogates the cGAS/STING-Mediated Cytosolic DNA-Sensing Pathway via Its Virion Host Shutoff Protein, UL41. Journal of virology 2017, 91(6).

13. Xu H, Su C, Pearson A, Mody C, Zheng C. Herpes Simplex Virus 1 UL24 Abrogates the DNA Sensing Signal Pathway by Inhibiting NF-KB Activation. Journal of virology 2017, 91(7).

14. You H, Zheng S, Huang Z, Lin Y, Shen Q, Zheng C: Herpes Simplex Virus 1 Tegument Protein UL46 Inhibits TANK-Binding Kinase 1-Mediated Signaling. mBio 2019, 10(3).

15. Ye R, Su C, Xu H, Zheng C. Herpes Simplex Virus 1 Ubiquitin-Specific Protease UL36 Abrogates NF-kB Activation in DNA Sensing Signal Pathway. Journal of virology 2017, 91(5).

16. You H, Lin Y, Lin F, Yang M, Li J, Zhang R, Huang Z, Shen Q, Tang R, Zheng C. $\beta$-Catenin Is Required for the cGAS/STING Signaling Pathway but Antagonized by the Herpes Simplex Virus 1 US3 Protein. Journal of virology 2020, 94(5).

17. Huang J, You H, Su C, Li Y, Chen S, Zheng C. Herpes Simplex Virus 1 Tegument Protein VP22 Abrogates cGAS/STING-Mediated Antiviral Innate Immunity. Journal of virology 2018, 92(15).

18. Wu J, Li W, Shao Y, Avey D, Fu B, Gillen J, Hand T, Ma S, Liu X, Miley W, et al. Inhibition of cGAS DNA Sensing by a Herpesvirus Virion Protein. Cell Host Microbe. 2015;18(3):333-44.

19. Zhang G, Chan B, Samarina N, Abere B, Weidner-Glunde M, Buch A, Pich A, Brinkmann M, Schulz T. Cytoplasmic isoforms of Kaposi sarcoma herpesvirus LANA recruit and antagonize the innate 
immune DNA sensor cGAS. Proc Natl Acad Sci USA. 2016;113(8):E1034-43.

20. Verrier E, Yim S, Heydmann L, El Saghire H, Bach C, Turon-Lagot V, Mailly L, Durand S, Lucifora J, Durantel D, et al. Hepatitis B Virus Evasion From Cyclic Guanosine Monophosphate-Adenosine Monophosphate Synthase Sensing in Human Hepatocytes. Hepatology. 2018;68(5):1695-709.

21. Lok A, McMahon B. Chronic hepatitis B. Hepatology. 2007;45(2):507-39.

22. Lee S, Cha E, Lim J, Kwon S, Kim D, Cho H, Han K. Structural characterization of an intrinsically unfolded mini-HBX protein from hepatitis B virus. Mol Cells. 2012;34(2):165-9.

23. Jiang $\mathrm{J}$, Tang $\mathrm{H}$. Mechanism of inhibiting type I interferon induction by hepatitis $B$ virus $X$ protein. Protein cell. 2010;1(12):1106-17.

24. Tao J, Zhang X, Jin J, Du X, Lian T, Yang J, Zhou X, Jiang Z, Su X. Nonspecific DNA Binding of cGAS $N$ Terminus Promotes cGAS Activation. Journal of immunology (Baltimore Md: 1950). 2017;198(9):3627-36.

25. Wang X, Li Y, Mao A, Li C, Li Y, Tien P. Hepatitis B virus $X$ protein suppresses virus-triggered IRF3 activation and IFN-beta induction by disrupting the VISA-associated complex. Cell Mol Immunol. 2010;7(5):341-8.

26. Chen M, Meng Q, Qin Y, Liang P, Tan P, He L, Zhou Y, Chen Y, Huang J, Wang R, et al. TRIM14 Inhibits cGAS Degradation Mediated by Selective Autophagy Receptor p62 to Promote Innate Immune Responses. Molecular cell. 2016;64(1):105-19.

27. Park S, Rehermann B. Immune responses to HCV and other hepatitis viruses. Immunity. 2014;40(1):13-24.

28. Xiong M, Wang S, Wang Y, Ran Y. The Regulation of cGAS. Virol Sin. 2018;33(2):117-24.

29. Gao P, Ascano M, Wu Y, Barchet W, Gaffney B, Zillinger T, Serganov A, Liu Y, Jones R, Hartmann G, et al. Cyclic $\left[G\left(2^{\prime}, 5^{\prime}\right) p A\left(3^{\prime}, 5^{\prime}\right) p\right]$ is the metazoan second messenger produced by DNA-activated cyclic GMP-AMP synthase. Cell. 2013;153(5):1094-107.

30. Zhao B, Xu P, Rowlett C, Jing T, Shinde O, Lei Y, West A, Liu W, Li P. The molecular basis of tight nuclear tethering and inactivation of cGAS. Nature. 2020;587(7835):673-7.

31. Chen H, Chen H, Zhang J, Wang Y, Simoneau A, Yang H, Levine A, Zou L, Chen Z, Lan L. cGAS suppresses genomic instability as a decelerator of replication forks. Science advances 2020, 6(42).

\section{Tables}




\begin{tabular}{|ll|}
\hline \multicolumn{2}{|l|}{ Table 1. Primers for qRT-PCR } \\
\hline primer & sequence \\
\hline cGAS Forward & 5'AAGCTCCGGGCGGTTTTGGA 3' \\
\hline cGAS Reverse & 5'AGGTGCAGAAATCTTCACGTGCTC3' \\
\hline STING Forward & 5'GTGGCTTGAGGGGAACCCGC3' \\
\hline STING Reverse & 5'GGCTGGAGTGGGGCATCTTCT3' \\
\hline IFN- $\beta$ Forward & 5'AGGACAGGATGAACTTTGAC3' \\
\hline IFN- $\beta$ Reverse & 5'TGATAGACATTAGCCAGGAG3' \\
\hline ISG56 Forward & 5'TCTCAGAGGAGCCTGGCTAA3' \\
\hline ISG56 Reverse & 5'TGACATCTCAATTGCTCCAG 3' \\
\hline ISG54 Forward & 5'GGAGCAGATTCTGAGGCTTTGC3' \\
\hline ISG54 Reverse & 5'GGATGAGGCTTCCAGACTCCAA3' \\
\hline
\end{tabular}

Table2. Abbreviations 


\begin{tabular}{|c|}
\hline Full name : Abbreviations \\
\hline cGASDCyclic GMP-AMP synthase \\
\hline HBVGHepatitis B virus \\
\hline HBXDHepatitis B virus $X$ protein \\
\hline PRRs $\square$ Pathogen recognition receptors \\
\hline IFN-IDType I interferon \\
\hline IRF3DInterferon regulatory factor-3 \\
\hline ISGsDIFN stimulated genes \\
\hline HSV-10Herpes simplex virus 1 \\
\hline DENVIDengue virus \\
\hline KSHVDKaposi's sarcoma-associated herpesvirus \\
\hline LANADLatency-associated nuclear antigen \\
\hline DMEMDDulbecco's modified Eagle's medium \\
\hline pRL-TKDRenilla luciferase \\
\hline DOCDDeoxycholate \\
\hline DLRDDual-luciferase reporter \\
\hline dsDNA[D ouble-stranded DNA \\
\hline K48DLysine 48 \\
\hline LC3ロmicrotubule-associated protein-Light-chain 3 \\
\hline pcDNA-HBV1.3DHBV 1.3-fold genome plasmid \\
\hline IFN- $\beta$ IInterferon beta \\
\hline TBK10Tank-binding kinase 1 \\
\hline 3-MAD3-methyladenine \\
\hline ISGロInterferon-inducible Protein 6-16 \\
\hline ISG56IIFN-stimulated gene 56 \\
\hline ISG54DIFN-stimulated gene 54 \\
\hline Western-Blot: WB \\
\hline
\end{tabular}



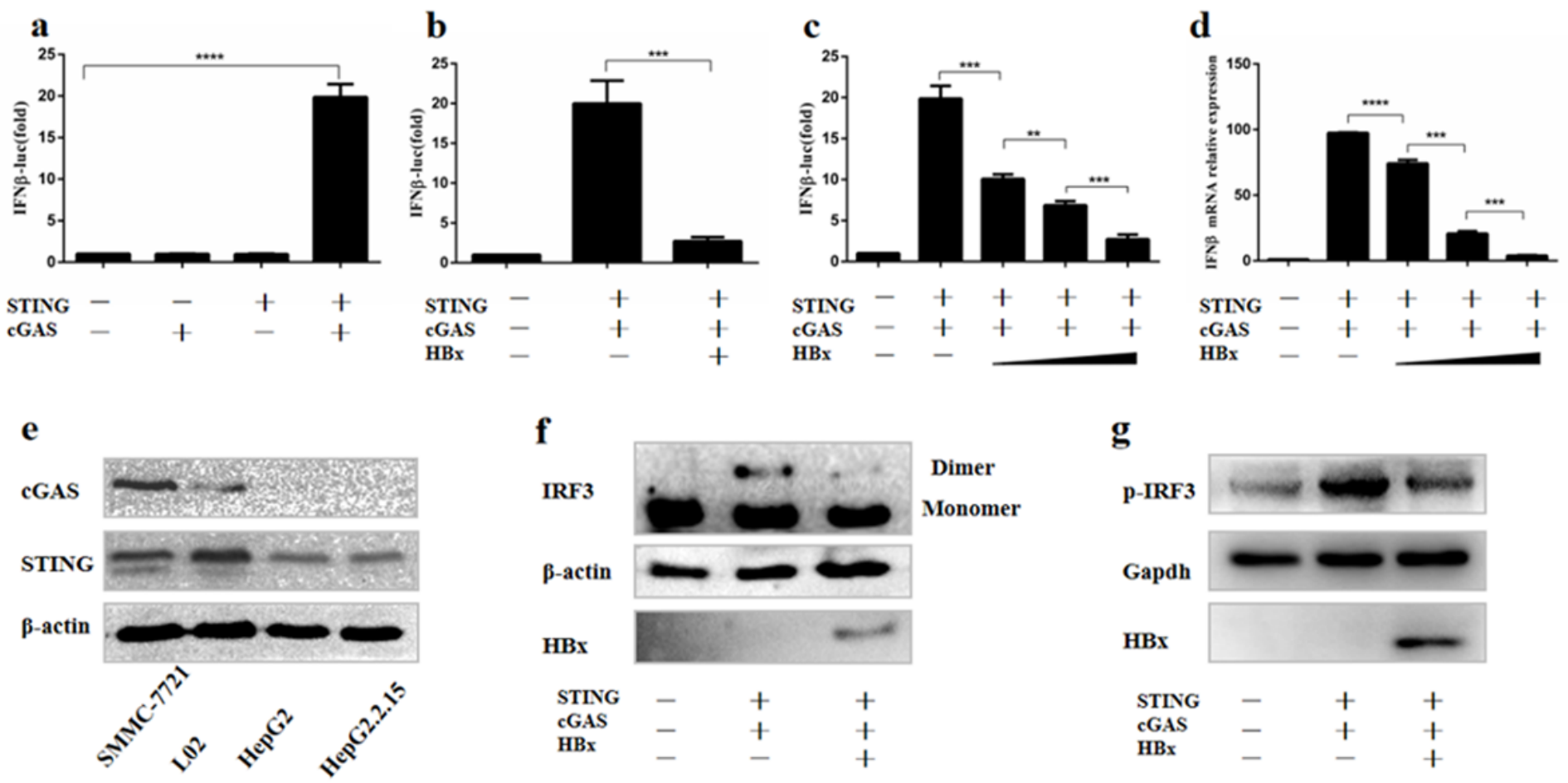

Figure 1

Hepatitis B virus X protein is a Negative Regulator of cGAS-Induced IFN-I- $\beta$ Signaling. (a-c) HEK293T cells were transfected with IFN-I- $\beta$ luciferase reporter and expression plasmids as indicated, and luciferase activity was assayed $24 \mathrm{~h}$ after transfection. The relative luciferase activity was expressed as arbitrary units by normalizing firefly luciferase activity to Renilla luciferase activity. (d) HEK293T cells were transfected with expression plasmids as indicated, at $24 \mathrm{~h}$ posttransfection, cells were harvested and subjected to qRT-PCR analysis. (e) Western blot (WB) analysis of cGAS, STING in several human hepatic cell lines. (f, g) HEK293T cells were cotransfected with cGAS and STING, along with empty vector or HBxFlag plasmid, Western blot analysis of IRF3 dimerization, and phosphorylation. 
$\mathbf{a}$
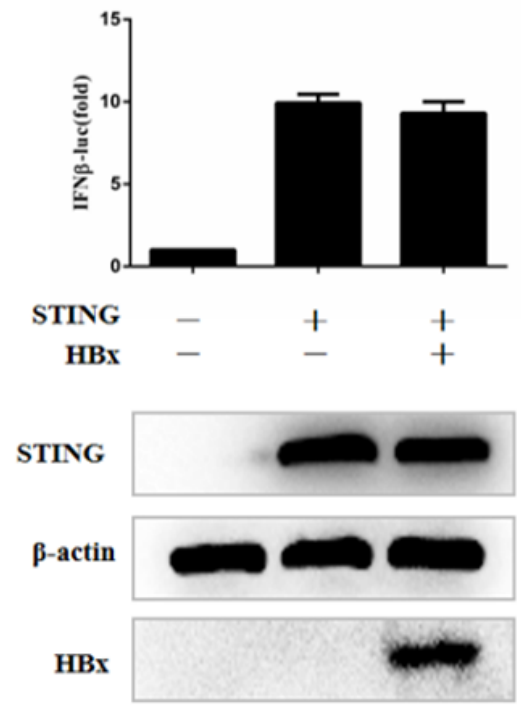

b
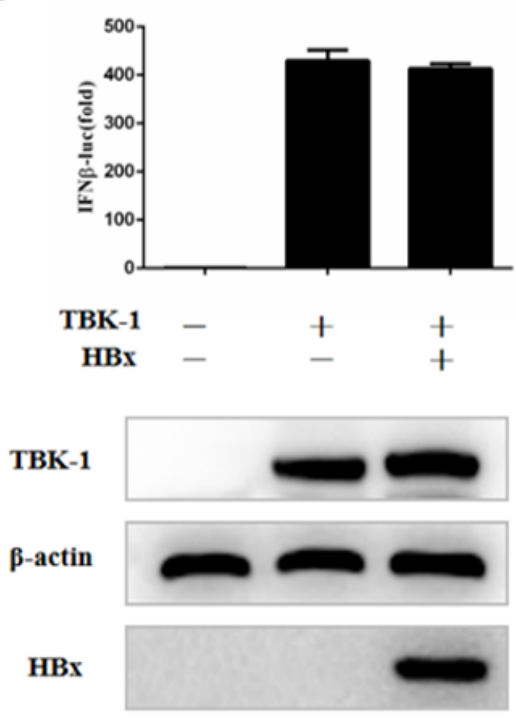

c

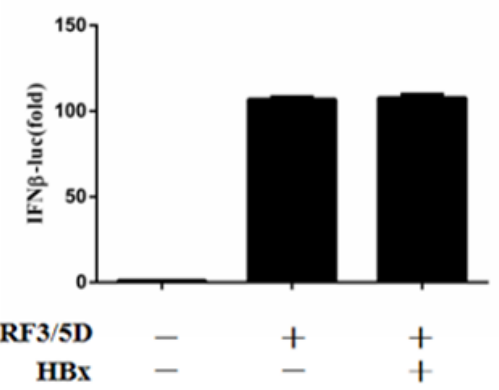

IRF3/5D

Gapdh

HBx

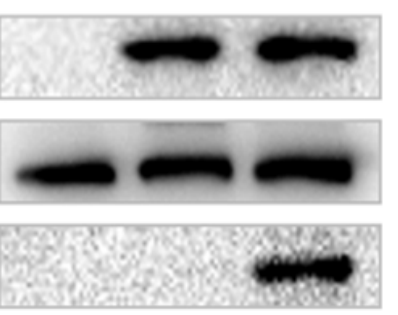

\section{Figure 2}

HBx inhibits the IFN-I- $\beta$ signaling pathway at the level upstream of STING. (a-c) HEK293T cells were cotransfected with expression plasmids as indicated, and cells were harvested $24 \mathrm{~h}$ after transfection and subjected to DLR assay. The expression of STING, IRF3/5D, TBK1, and HBx was analyzed by WB analysis. The data represent results from one of the triplicate experiments. Error bars represent standard deviations of three independent experiments. 
a

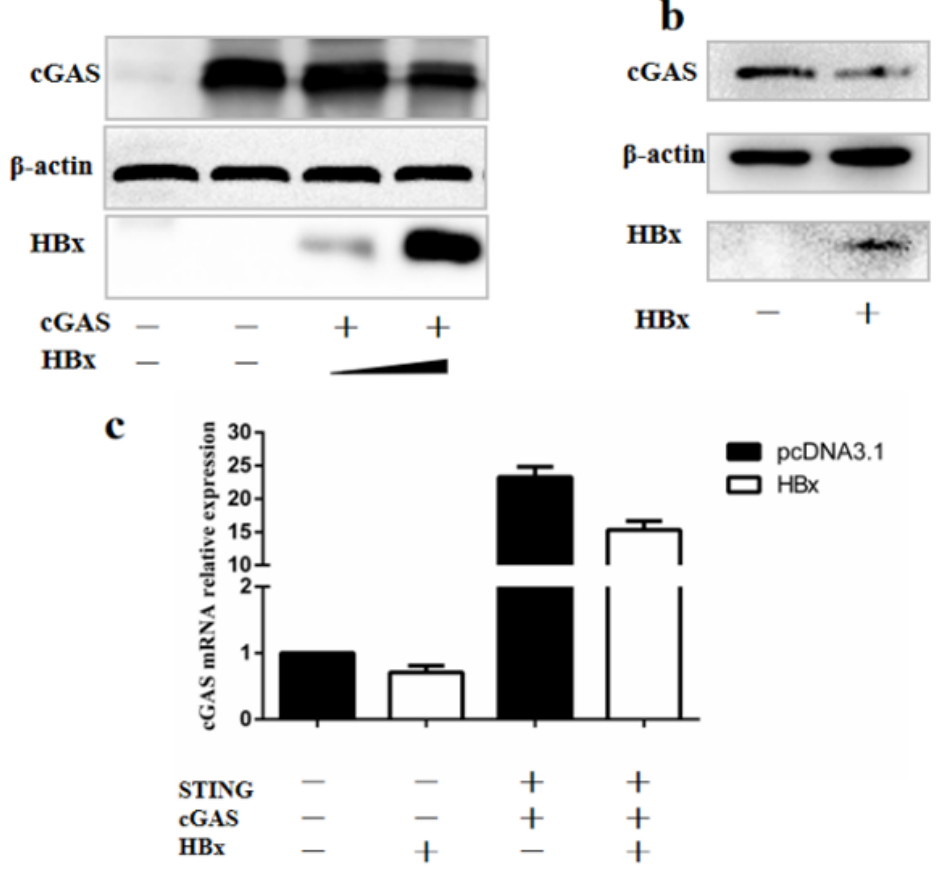

Figure 3

HBx down-regulates the cGAS expression and function. (a) HEK293T cells were transfected with expression plasmids as indicated, and then cells were harvested $24 \mathrm{~h}$ postinfection and subjected to WB analysis. (b) SMMC-7721 cells were transfected with expression plasmids as indicated at $18 \mathrm{~h}$ posttransfection, and cells were harvested and subjected to qRT-PCR analysis. The data represent results from one of the triplicate experiments. Error bars represent the SDs of three independent experiments. 
a

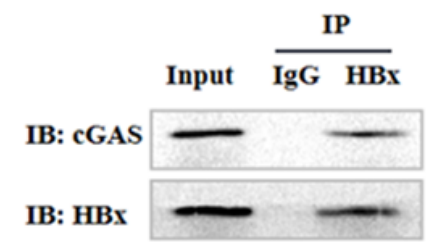

b

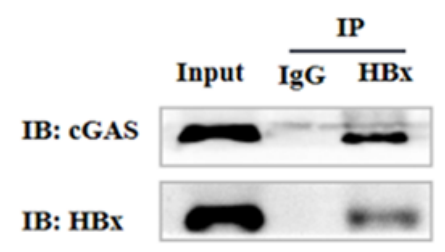

c

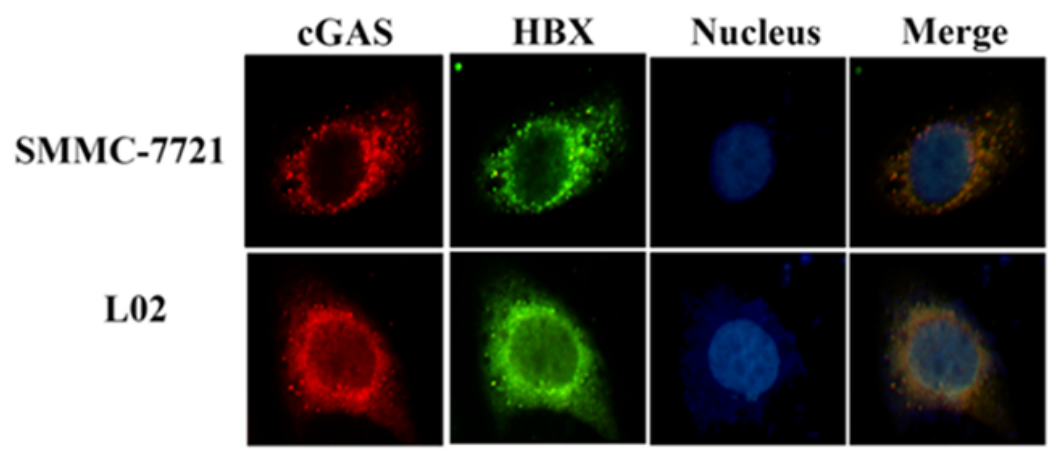

\section{Figure 4}

HBx binds to and interacts with cGAS. $(a, b)$ SMMC-7721 cells were transfected with an empty vector or $\mathrm{HBx}$-Flag plasmid. At 24h posttransfection, cells were harvested, and obtain total protein was. We used the IgG and anti-HBx or anti-cGAS antibody to pull the protein, and the interaction was detected by western blot analysis. (c) SMMC-7721 and L02 cells were transfected with an empty vector or HBx-Flag plasmid. At 24h posttransfection, stain with fluorescent antibody as indicated and observed by fluorescence microscope. 
a
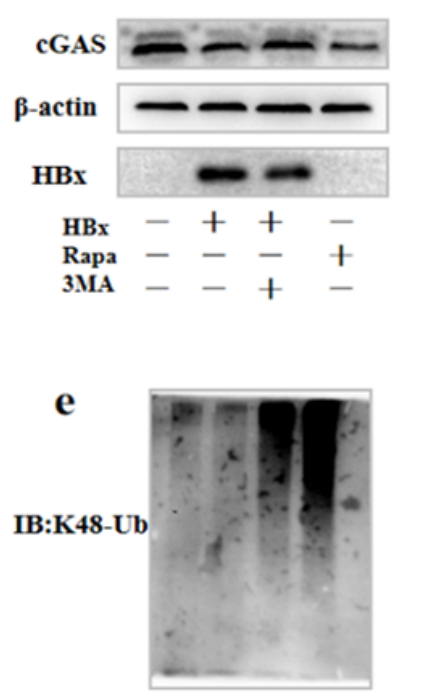

cGAS

$\beta$-actin

HBx

$\mathrm{HBx}-+-+$

MGl32 - ++ b

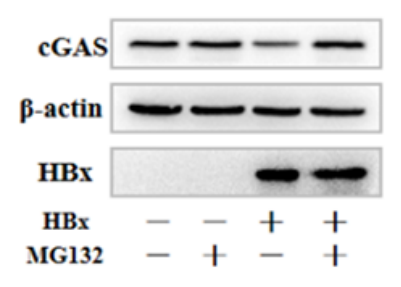

$\mathbf{f}$

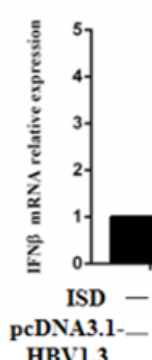

HBVi.3

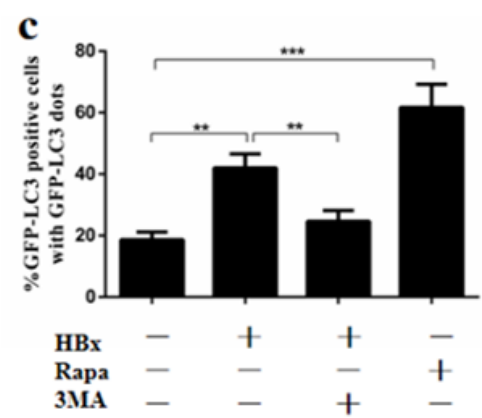

g

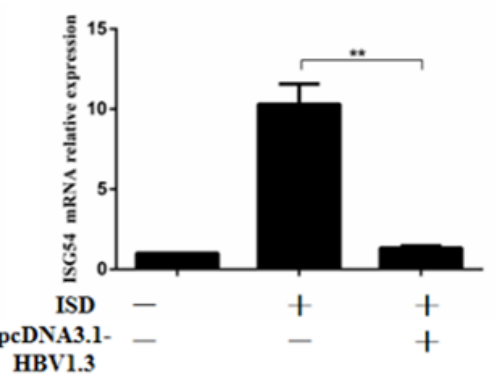

d

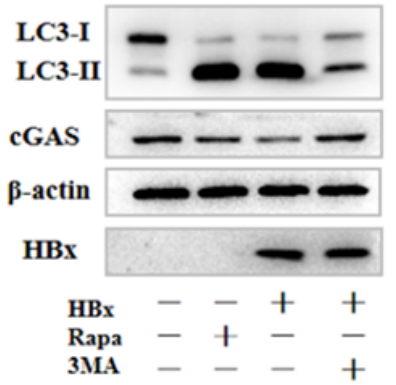

h

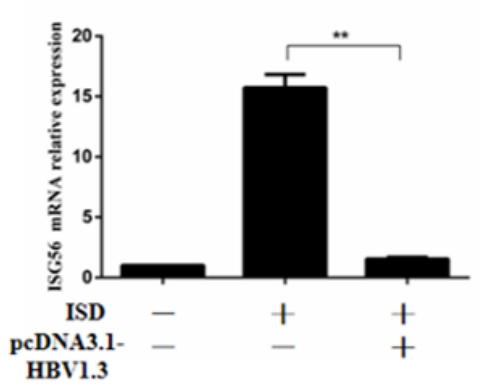

Figure 5

HBx promotes ubiquitination and autophagy degradation of cGAS. (a-b) SMMC-7721 cells were transfected with an empty vector or HBx-Flag plasmid. At $12 \mathrm{~h}$ posttransfection, treated with Rapamycin or 3-MA or MG132 (10 mM) for $12 \mathrm{~h}$, dimethyl sulfoxide (DMSO) was used for controls. Cells were harvested and subjected to WB analysis. (c, d) SMMC-7721 cells were transfected with pGFP-LC3 and empty vector or HBX- Flag plasmid. At $12 \mathrm{~h}$ posttransfection, treated with Rapamycin or 3MA or MG132 $(10 \mathrm{mM})$ for $12 \mathrm{~h}$, dimethyl sulfoxide (DMSO) was used for controls. Moreover, autophagic dots were observed by a fluorescence microscope. Quantitation of the autophagic cells in SMMC-7721. Cells with more than three fluorescent particles are considered autophagy-activated cells, meawhile冈the cells were harvested and subjected to WB analysis. (e) SMMC-7721 cells were transfected with an empty vector or HBx- Flag plasmid. At $12 \mathrm{~h}$ posttransfection, cells were harvested and subjected to WB analysis. (f-h) L02 were transfected with ISD, along with empty vector or HBV1.3 plasmid, luciferase activity was assayed 12 $\mathrm{h}$ after transfection. 